Preface

\title{
Acquired Platelet Dysfunction-Laboratory and Clinical Implications
}

\author{
Anne-Mette Hvas, MD, $\mathrm{PhD}^{1}$ Julie B. Larsen, MD, $\mathrm{PhD}^{1}$ Leonardo Pasalic, MBBS, $\mathrm{PhD}^{2,3}$ \\ ${ }^{1}$ Thrombosis and Hemostasis Research Unit, Department of Clinical \\ Biochemistry, Aarhus University Hospital, Aarhus, Denmark \\ ${ }^{2}$ Department of Laboratory Hematology, Institute of Clinical \\ Pathology and Medical Research,Westmead Hospital, Westmead, \\ New South Wales, Australia \\ ${ }^{3}$ Sydney Centres for Thrombosis and Hemostasis, Westmead, \\ New South Wales, Australia
}

Semin Thromb Hemost 2020;46:235-237.

In contrast to congenital platelet disorders, which are rare, acquired thrombocytopenia and platelet dysfunction are both frequently encountered in clinical practice. The usual clinical manifestations are mucosal bleeding, epistaxis, and superficial epidermal bleeding, generally of modest extent. However, when the patient is exposed to a hemostatic challenge (e.g., surgery), impaired platelet dysfunction or thrombocytopenia might impose a substantial bleeding risk. The main cause of platelet dysfunction is the use of antiplatelet drugs, but more importantly, systemic disorders have long been recognized as contributors to platelet dysfunction and/or thrombocytopenia and thereby increased bleeding risk. In this issue of Seminars of Thrombosis and Hemostasis, our invited contributors share their expertise on thrombocytopenia and acquired platelet dysfunction within several very different clinical entities, outside the use of antiplatelet drugs. Strategies are presented on how to diagnose and treat patients with thrombocytopenia and/or acquired platelet dysfunction to optimize patient care.

As the first contribution in the present issue, Lippi et al discuss the frequently asked question regarding platelet transfusion thresholds: How low can we go in respect to platelet counts? ${ }^{1}$ The review provides an overview of current guidelines and recommendations for platelet transfusion thresholds across different clinical conditions such as surgery (minor or major), neurosurgery, lumbar puncture, and platelet function defects. Moreover, the current analytical performance of platelet counting employing fully automated hematological analyzers is critically addressed followed by potential solutions to overcome the current shortcomings of automated platelet counting.

Address for correspondence Anne-Mette Hvas, MD, PhD, Thrombosis and Hemostasis Research Unit, Department of Clinical Biochemistry, Aarhus University Hospital, Palle Juul-Jensens Boulevard 99, DK - 8200 Aarhus N, Denmark (e-mail: am.hvas@dadlnet.dk).

In continuation, Estcourt provides a comprehensive review of the handling of thrombocytopenia in surgery and neuroaxial anesthesia. ${ }^{2}$ Evidence is presented for the use of different platelet count thresholds prior to invasive procedures and surgery, taking into account the difficult balance of the benefit-risk ratio for the use of platelet transfusions. Beyond minor surgery, the review focuses on four procedures that are common in patients with thrombocytopenia: central venous catheter insertion, liver biopsy, lumbar punctures, and insertion of epidural catheters. Finally, the lack of evidence for platelet transfusion thresholds in major surgery during thrombocytopenia is highlighted.

Thrombocytopenia is a common finding and potential complication during pregnancy. The approach to the diagnosis and management of thrombocytopenia in pregnancy and labor is thoroughly discussed by Fogerty. ${ }^{3}$ As highlighted in the review, establishing the etiology is essential. This ranges from (1) the, most often, benign gestational thrombocytopenia to (2) pregnancy-specific thrombocytopenia as preeclampsia, or (3) pregnancy-triggered thrombocytopenia such as thrombotic thrombocytopenic purpura (TTP), to (4) thrombocytopenia being related to other coexisting medical conditions such as liver disease or infections. Pathophysiological mechanisms of the most common etiologies are presented, as well as the associated management ranging from observation to urgent fetal delivery depending on the etiology.

Drug-induced thrombocytopenia deserves special attention with a prevalence of around $25 \%$ among critically ill patients. ${ }^{4}$ As highlighted by Danese et al in the included review on drug-induced thrombocytopenia, an early and accurate diagnosis of this entity is crucial for reversing an

Copyright (c) 2020 by Thieme Medical Publishers, Inc., 333 Seventh Avenue, New York, NY 10001, USA. Tel: +1(212) 760-0888.
DOI https://doi.org/ 10.1055/s-0040-1708826. ISSN 0094-6176.
Dysfunction-Laboratory an

Clinical Implications; Guest

Editors: Anne-Mette Hvas, MD, PhD,

Julie Brogaard Larsen, MD, PhD, and Leonardo Pasalic, MBBS, PhD. 
otherwise unfavorable clinical outcome. ${ }^{5}$ In light of this, the underlying pathogenetic mechanisms are comprehensively reviewed, including reduced platelet production due to bone marrow suppression or megakaryocyte impairment and accelerated clearance of platelets from the circulation. As detailed by the authors, the latter form can be sustained by nonimmune mechanisms, as found in thrombotic microangiopathy, due to a direct dose-related toxic effect after exposure to several drugs, among others chemotherapeutics and immunosuppressive agents; immune-mediated druginduced thrombocytopenia by drug-dependent antibodies; and autoimmune mechanisms mediated by drug-independent antibodies, as seen in heparin-induced thrombocytopenia. As early diagnosis of drug-induced thrombocytopenia is crucial, a section on laboratory testing for drug-induced thrombocytopenia is also presented. ${ }^{5}$

Al-Samkari and Kuter then present a comprehensive review on modern approaches to diagnosis, as well as new and old treatment options of immune thrombocytopenia (ITP) in adults. ${ }^{6}$ As identified by the authors, the diagnosis of ITP has not essentially changed in the past decade. As no reliable biomarker or gold-standard diagnostic test exists for ITP, the diagnosis still remains clinical, with exclusion of other causes of thrombocytopenia. As pointed out in the review, treatment of ITP has advanced considerably in the past decade, with the introduction of thrombopoietin receptor agonists that augment platelet production, the tyrosine kinase inhibitor fostamatinib, which was recently approved by the U.S. Food and Drug Administration, as well as other immunosuppressive agents. As presented in the review, numerous additional agents are currently under development for ITP treatment, and thereby continued advances are likely moving forward.

Immune-mediated TTP is a rare but potentially lethal condition. In a comprehensive narrative review, Blennerhassettet al first set the scene with a historical perspective of recognition of the disease. ${ }^{7}$ In continuation, the pathophysiology, epidemiology, and the essential clinical features are summarized. The diagnostic approach including discussion of essential laboratory tests are included, as well as both current and novel targeted therapies. The authors stress the importance of maintaining clinician vigilance in the initial detection of the disease, management of refractory disease, and prediction and prevention of TTP relapse.

During the past decade, an increasing amount of research has focused on the role of platelets beyond hemostasis. The role of platelets in infections, both bacterial and viral, is extensively reviewed by Page and Pretorius. ${ }^{8}$ First, the contribution of platelets to the immune response is reviewed and illustrated in detail. Second, platelet interactions with bacteria are presented, including platelet receptors in bacterial pathogen sensing and how bacterial products affect platelet function. Finally, platelet interactions with viruses are reviewed. The authors conclude that during infection, an onslaught of inflammatory and pathogen-derived stimuli can stimulate platelets and thereby lead to inappropriate activation, immunological destruction, and sequestration of platelets. In the context of a dysregulated host response to infection, platelets can experience overwhelming activation and, consequently, consumption, and this generic large-scale mechanism for platelet depletion may cause significant health issues.

Sepsis is associated with high morbidity and mortality, and short-term mortality remains high despite relevant treatment. To investigate improved tools for monitoring these patients, Thorup et al summarize the evidence for a potential association between an increased number of circulating immature platelets and disease severity and mortality in patients with sepsis and septic shock. ${ }^{9}$ Notably, the systematic review confirms the hypothesis of an association between an increased level of circulation immature platelets and disease severity and mortality in sepsis. This increased level of circulating immature platelets might be due to an increased platelet turnover subsequent to increased platelet consumption during sepsis. As a complication, the increased number of immature platelets might contribute to microthrombus formation and organ failure and thereby enhance the risk of increased severity and mortality in sepsis. As suggested by the authors, markers of immature platelets could potentially improve the monitoring of patients with sepsis or septic shock by being used together with the Sequential Organ Failure Assessment, routinely used in these patients.

Cancer patients face an increased risk of bleeding both due to the cancer itself and due to the antineoplastic treatment often causing thrombocytopenia. ${ }^{10}$ In the systematic review by Larsen et al, current evidence is presented for the role of platelets in terms of thrombocytopenia and/or impaired platelet function in cancer patients. ${ }^{11}$ The association between platelet count and bleeding was found to be most pronounced at platelet counts $\leq 10 \times 10^{9} / \mathrm{L}$. This was by far most evident for hematological malignancy, whereas more evidence is needed as regards patients with solid tumors. Until now, rather few studies have investigated platelet function in cancer patients, but, overall, a reduced platelet function was found to be significantly associated with bleeding risk in these patients. The present review points to the fact that further investigations of platelet function and the potential role of platelet function analysis are warranted for the assessment of bleeding risk in cancer patients.

Paradoxically, patients with advanced chronic kidney disease have both increased risk of both thromboembolic disease and bleeding. As reviewed by Lutz and Jurk, ${ }^{12}$ platelets play a pivotal role in the pathogenesis of both entities. As part of the section on the role of platelet activation in thromboembolic risk, signaling molecules and uremic toxins are explored, as well as platelet-platelet and platelet-leukocyte interaction, the increased expression of anionic phospholipids on platelets, and elevated levels of platelet-derived microparticles in patients with chronic kidney disease. This is followed by a section on platelet dysfunction and bleeding risk due to, for example, uremic toxins, anemia, and certain antibiotics often administered to patients with chronic kidney disease. As detailed by the authors, investigations of platelet function are challenging due to the complexity of platelet function alterations. Finally, recommendations are provided for therapy 
of thrombotic disorders in patients with chronic kidney disease as well as possible therapeutic agents to be used to treat bleeding in these patients.

Extracorporeal membrane oxygenation (ECMO) can be lifesaving support in neonates, children, and adults with respiratory and/or cardiac failure refractory to conventional treatment. However, hemorrhagic and thrombotic complications are common, resulting in considerable morbidity or mortality. ${ }^{13}$ Neonatal and pediatric patients can be particularly vulnerable to blood loss, as critical volumes are smaller, and children and neonates, in particular, differ from adults regarding both platelet count and function. In the present issue, Cashen et al provide an overview of hemostatic challenges during pediatric ECMO with a focus on platelet count and function, and the association with bleeding risk. ${ }^{14}$ The review points to the important fact that platelet function testing is very challenging in neonatal and pediatric patients due to the relatively large volume of blood needed for this analysis and the lack of standardization of platelet function tests, especially in children. As a consequence, a paucity of evidence exists on platelet function during pediatric ECMO support, and platelet counts are most often the measure used to monitor the role of platelets and judge the need for platelet transfusions during ECMO. Finally, Cashen et al discuss future needs for optimization of patient management during ECMO in light of the critical need of reducing the risk of bleeding and thromboembolism during pediatric ECMO support. ${ }^{14}$

Intraventricular hemorrhage is a severe complication of premature birth and affects up to $22 \%$ of extremely low birth weight neonates. ${ }^{15}$ Grevsen et al present a systematic review and meta-analysis of the role of platelet counts and other platelet indices in premature neonates with intraventricular hemorrhage. ${ }^{16}$ Moreover, current knowledge on the impact of secondary hemostasis and fibrinolysis is highlighted. One major finding presented by the authors is that neonates with platelet counts $<100 \times 10^{9} / \mathrm{L}$ during the first week of life have an increased incidence of intraventricular hemorrhage. Results on the impact of secondary hemostasis are conflicting, but, overall, no consistent difference is found in secondary hemostasis parameters between preterm neonates with and without intraventricular hemorrhage. Fibrinolysis is only sparsely investigated; thus, it is not possible to conclude on the role of fibrinolysis in intraventricular hemorrhage in preterm neonates. Whether reduced platelet function is associated with a risk of intraventricular hemorrhage in premature neonates also remains to be investigated.

In summary, this issue of Seminars in Thrombosis $\mathcal{E}$ Hemostasis highlights several clinical conditions complicated by thrombocytopenia and/or acquired platelet dysfunction that mostly implies increased bleeding risk but also, for some patients, an increased thromboembolic risk. The reviews included also point to the research needed to further improve current knowledge within this subject area. We hope readers will enjoy the content and that this leads to a further discussion of the variety of topics presented herein.

At the time of compiling this issue on acquired platelet dysfunction we recognize the growing worldwide crisis and pandemic caused by COVID-19. In response, the senior editors of STH have started to compile a series of Commentaries related to COVID-19 and within their specific area of expertise. The first of these is also included in this issue of STH. ${ }^{17}$ We intend further contributions in subsequent issues of the journal.

\section{Conflicts of Interest}

None.

\section{References}

1 Lippi G, Favaloro EJ, Buoro S. Platelet transfusion thresholds: how low can we go in respect to platelet counting? Semin Thromb Hemost 2020;46(03):238-244

2 Escourt LJ. Thrombocytopenia in surgery and neuroaxial anesthesia. Semin Thromb Hemost 2020;46(03):245-255

3 Fogerty AE. Thrombocytopenia in pregnancy: approach to diagnosis and management. Semin Thromb Hemost 2020;46(03):256-263

4 Kenney B, Stack G. Drug-induced thrombocytopenia. Arch Pathol Lab Med 2009;133(02):309-314

5 Danese E, Montagnana M, Favaloro EJ, Lippi G. Drug-induced thrombocytopenia (DIT): mechanisms and laboratory diagnostics. Semin Thromb Hemost 2020;46(03):264-274

6 Al-Samkari H, Kuter DJ. Immune thrombocytopenia in adults: Modern approaches to diagnosis and treatment. Semin Thromb Hemost 2020;46(03):275-288

7 Blennerhassett R, Curnow J, Pasalic L. Immune-mediated thrombotic thrombocytopenic purpura: a narrative review of diagnosis and treatment in adults. Semin Thromb Hemost 2020;46(03):289-301

8 Page MJ, Pretorius E. A champion of host defence: a generic largescale cause for platelet dysfunction and depletion in infection. Semin Thromb Hemost 2020;46(03):302-319

9 Thorup CV, Christensen S, Hvas AM. Immature platelets as a predictor of disease severity and mortality in sepsis and septic shock: a systematic review. Semin Thromb Hemost 2020;46(03): 320-327

10 Weycker D, Hatfield M, Grossman A, et al. Risk and consequences of chemotherapy-induced thrombocytopenia in US clinical practice. BMC Cancer 2019;19(01):151. doi: 10.1186/s12885-019-5354-5

11 Larsen JB, Hojbjerg JA, Hvas AM. The role of platelets in cancerrelated bleeding risk: a systematic review. Semin Thromb Hemost 2020;46(03):328-341

12 Lutz J, Jurk K. Platelets in advanced chronic kidney disease: two sides of the coin. Semin Thromb Hemost 2020;46(03): 342-356

13 Dalton HJ, Reeder R, Garcia-Filion P, et al; Eunice Kennedy Shriver National Institute of Child Health and Human Development Collaborative Pediatric Critical Care Research Network. Factors associated with bleeding and thrombosis in children receiving extracorporeal membrane oxygenation. Am J Respir Crit Care Med 2017;196(06):762-771

14 Cashen K, Meert K, Dalton HJ. Platelet count and function during pediatric extracorporeal membrane oxygenation. Semin Thromb Hemost 2020;46(03):357-365

15 Ancel PY, Goffinet F, Kuhn P, et al; EPIPAGE-2 Writing Group. Survival and morbidity of preterm children born at 22 through 34 weeks' gestation in France in 2011: results of the EPIPAGE-2 cohort study. JAMA Pediatr 2015;169(03):230-238

16 Grevsen AK, Hviid CVB, Hansen AK, Hvas AM. The role of platelets in premature neonates with intraventicular hemorrhage: a systematic review and meta-analysis. Semin Thromb Hemost 2020; 46(03):366-378

17 Favaloro EJ, Lippi G. Commentary: Recommendations for Minimal Laboratory Testing Panels in Patients with COVID-19: Potential for Prognostic Monitoring. Semin Thromb Hemost 2020;46(03): 379-382 